\section{Isquemia arterial aguda causada por embolismo paroadójico}

\section{Paradoxical embolism causing acute arterial ischemia}

\section{Sr. Director:}

La embolia paradójica (EP) supone el paso de un trombo del territorio venoso al sistema arterial, a través de una comunicación derecha-izquierda que causa una obstrucción arterial aguda ${ }^{1}$. La alta prevalencia de trombosis venosa profunda y la existencia de foramen oval permeable (FOP) en un porcentaje elevado de población, hace que esta situación deba tenerse en cuenta en casos atípicos de isquemia aguda arterial ${ }^{2}$.

Presentamos el caso de una mujer de 59 años con historia de trastornos vasculares periféricos que consulta en Urgencias por debilidad y dolor en miembros inferiores, acompañado de sensación disneica y taquipnea. En la analítica destaca hipoxemia e hipocapnia y en electrocardiograma se objetiva taquicardia sinusal. Se solicita ecocardiografía en la que se encuentra ventrículo y aurícula derechos dilatados, calculando una presión en arteria pulmonar (PAP) de $60 \mathrm{mmHg}$. Se concluye la presencia de hipertensión pulmonar severa $(65-70 \mathrm{mmHg})$ explicada por probable tromboembolismo pulmonar.

Tras ingresar en UCl se efectúa fibrinolisis con rtPA (30 mg iv en 5 min más $70 \mathrm{mg}$ iv en $90 \mathrm{~min}$ ), manteniéndose después una perfusión de heparina sódica como anticoagulación. Al mejorar la situación clínica se realiza ecocardiografía, observándose reducción de la PAP $(55 \mathrm{mmHg})$, con cavidades aún dilatadas. Persiste la clínica de impotencia funcional y dolor en miembros inferiores, detectando ausencia de pulsos periféricos. Se decide la realización de arteriografía, informada como oclusión aguda en ambas arterias femorales, por lo que se plantea intervención quirúrgica urgente, practicándose embolectomía transfemoral bilateral por Cirugía Vascular, sin incidencias. Sospechando la posibilidad de una EP solicitamos ecocardiografía transesofágica: ausencia de trombos; FOP $(6-7 \mathrm{~mm})$ con flujo derechaizquierda. Finalmente la evolución fue satisfactoria. Se decidió mantener anticoagulación permanente, quedando pendiente una solución quirúrgica definitiva del foramen.

La EP supone un $2 \%$ de todos los fenómenos embolígenos arteriales. Sin embargo, la alta prevalencia del FOP (20-25\% en población general y 35\% en estudios autópsicos) justifica que deba ser considerada una posibilidad diagnóstica $^{3}$. Suele originarse a través del FOP, aunque en algunas ocasiones puede deberse a shunts derechaizquierda extracardíacos (malformaciones arteriovenosas pulmonares o fístulas arteriovenosas periféricas). La persistencia de FOP puede estar causada por motivos embriológicos, o por causas secundarias que aumentan la presión sobre aurícula derecha (tromboembolismo pulmonar, enfermedad pulmonar obstructiva crónica, hipertensión pulmonar primaria, ventilación mecánica o maniobra de Valsalva).

La ecocardiografía transesofágica es la prueba más sensible para detectar el FOP, utilizando como contraste una solución salina agitada. Antecedentes de episodios previos de trombosis venosa profunda o estados de hipercoagulabilidad justifican la sospecha diagnóstica, pero sólo se puede hacer un diagnóstico definitivo al observar de forma directa el paso del trombo a través del FOP mediante ecocardiografía. Ante la baja prevalencia de EP, son pocos los estudios que han profundizado en el tratamiento más adecuado. La resolución de la embolia, ya sea con tratamiento médico o quirúrgico, debe ser el paso inicial. Se debe prevenir su recidiva mediante terapias antiagregantes y anticoagulantes. $\mathrm{El}$ primer año es de alto riesgo de recurrencia y, por tanto, se aconseja una terapia anticoagulante, así como el cierre del foramen oval permeable ${ }^{4}$. Las distintas posibilidades de cierre del foramen oval incluyen el acceso quirúrgico mediante toracotomía abierta o el acceso percutáneo, aconsejado por los últimos estudios, dados sus satisfactorios resultados ${ }^{5}$.

En definitiva, la EP es una situación que debe tenerse en cuenta dentro de los fenómenos embolígenos arteriales de origen desconocido. En la actualidad no se dispone de una estrategia definida ante dicho evento y debe plantearse desde una perspectiva individual, teniendo en cuenta los factores de riesgo, la etiología del caso y el síndrome clínico presentado.

\section{Bibliografía}

1. Rakhit RD. Case 2: Patent foramen ovale and paradoxical embolism. Heart. 2003;89:1362.

2. Cramer SC. Patent Foramen Ovale and its Relationship to Stroke. Cardiol Clin. 2008;23:7-11.

3. Cheng TO. Impending Paradoxical Embolism. Circulation. 2000;101(23):e226.

4. ACC/AHA Guidelines for the Management of Adults With Congenital Heart Disease. J Am Coll Cardiol. 2008;52:e1-21.

5. Aslam F, Shirani J, Haque AA. Patent Foramen Ovale: Assessment, Clinical Significance and Therapeutic Options. South Med J. 2006;12:1367-72.

J. Revuelto Rey*, J.J. Egea Guerrero, R. Hinojosa Pérez y

R. Martín Bermúdez

Servicio de Medicina Intensiva, Hospital Universitario Virgen del Rocío, Sevilla, España

*Autor para correspondencia.

Correo electrónico: drjau@hotmail.com (J. Revuelto Rey). 